\title{
APPLICATION OF LAPLACE TRANSFORM HOMOTOPY PERTURBATION METHOD TO NONLINEAR PARTIAL DIFFERENTIAL EQUATIONS
}

\author{
Prem Kiran G. Bhadane ${ }^{1}$, V. H. Pradhan ${ }^{2}$ \\ ${ }^{1}$ Assistant Professor, Department of Applied Sciences, RCPIT, Maharashtra, India, \\ om.bhadane@rediffmail.com \\ ${ }^{2}$ Associate Professor, Department of Applied Mathematics and Humanities, SVNIT, Gujarat, India \\ pradhan65@yahoo.com
}

\begin{abstract}
The Laplace transform is widely used to solve linear ordinary and partial differential equations. Recently many researchers have applied the Laplace transform (LT) with variational iteration method (VIM), Adomian decomposition method (ADM) and homotopy perturbation method (HPM) to obtain the solution of nonlinear differential equations. In the present paper the solution of some nonlinear differential equations are obtained on combining the Laplace transform with homotopy perturbation method (LTHPM). The present method detects the solution without any discretization or restrictive assumptions and therefore reduces the numerical computations to a greater extent. The obtained solutions are compared with the available exact solutions and the solutions obtained by homotopy perturbation method (HPM). It shows that the method LTHPM has a good agreement with the exact solution in comparison with HPM.
\end{abstract}

Keywords: Laplace Transform, Partial Differential Equation (PDE) and Laplace Transform with Homotopy Perturbation Method.

\section{INTRODUCTION}

LTHPM is the method in which the Laplace transform (LT) is coupled with Homotopy perturbation method (HPM) for the approximate analytical solution of nonlinear differential equations. The present method is easy, efficient and widely useful for solving nonlinear differential equations. In HPM, a homotopy is constructed by introducing an embedding parameter $p \in[0,1]$. The HPM uses the small parameter $p$ and the solution is written as a power series. The changing process from zero to unity is called deformation in Topology. The nonlinear term can be decomposed as a power series in $p$ in terms of He's polynomials which can be generated by several means. The detailed description of this method can be found in [5]. An exhaustive survey on the application of HPM is available in the standard literature. [1]

Mostly various physical phenomena are governed by linear or nonlinear differential equations. Although several techniques like Laplace transform, Fourier transform and Sumudu transform are available for solving the linear differential equations, the solution of nonlinear differential equation can be obtained by combining these techniques with other analytical methods to handle the nonlinearities. Recently, many researchers have combined Laplace transform with HPM, ADM and VIM. In the present work the solution of nonlinear pde are obtained by combining LT with HPM.
Due to the capability of combining these two powerful tools, the LTHPM is found to an elegant method.

Ghorbani and Saberi-Nadjafi have used He's polynomials to handle the nonlinear term. LTHPM is simple and provides a closed form of the solution in most of the cases. The present approach has several advantages over other analytical methods like ADM, VIM and decrease the volume of calculation considerably and provide faster rate of convergence than HPM. In the next section two illustrations are taken whose exact solutions are already available in order to verify the reliability and efficiency of the LTHPM. The solutions obtained are same as exact solution. Hence, we conclude that amongst the existing numerical techniques LTHPM provides a nice refinement and can be widely use in various fields.

\section{Applications}

\subsection{Illustration I}

Consider,

$\frac{\partial^{2} u}{\partial t^{2}}-u \frac{\partial^{2} u}{\partial x^{2}}=1-\frac{x^{2}+t^{2}}{2}$

with the following conditions

$u(x, 0)=\frac{x^{2}}{2}, u_{t}(x, 0)=0$

Equation (1) has exact solution $u(x, t)=\frac{x^{2}+t^{2}}{2}$. 
According to LTHPM [3,4,6,7], first apply Laplace transform on equation (1),

$$
L\left\{\frac{\partial^{2} u}{\partial t^{2}}\right\}-L\left\{u \frac{\partial^{2} u}{\partial x^{2}}\right\}=L\left\{1-\frac{x^{2}+t^{2}}{2}\right\}
$$

Using the LT properties and with the help of specified initial condition (2), equation ( 3 ) can be written as

$$
L\{u(x, t)\}=\frac{x^{2}}{2 s}+\frac{1}{s^{3}}-\frac{x^{2}}{2 s^{3}}-\frac{1}{s^{5}}+\frac{1}{s^{2}} L\left[u \frac{\partial^{2} u}{\partial x^{2}}\right]
$$

Now, using inverse Laplace transform to equation (4),

$u(x, t)=\frac{x^{2}}{2}+\frac{t^{2}}{2}-\frac{x^{2} t^{2}}{4}-\frac{t^{4}}{24}+L^{-1}\left\{\frac{1}{s^{2}} L\left[u \frac{\partial^{2} u}{\partial x^{2}}\right]\right\}$

Applying the HPM, according to which $u$ is expanded into infinite series and nonlinear term is replaced by $\mathrm{He}$ 's polynomial

$$
\begin{aligned}
\sum_{n=0}^{\infty} p^{n} u_{n}(x, t)= & \frac{x^{2}}{2}+\frac{t^{2}}{2}-\frac{x^{2} t^{2}}{4}-\frac{t^{4}}{24} \\
& +p\left\{L^{-1}\left[\frac{1}{s^{2}} L\left[\sum_{n=0}^{\infty} p^{n} H_{n}(u)\right]\right]\right\}
\end{aligned}
$$

where $H_{n}(u)$ are He's polynomials.

Equate the coefficients of like powers of $p$ into equation (6), we have

$p^{0}: u_{0}(x, t)=\frac{x^{2}}{2}+\frac{t^{2}}{2}-\frac{x^{2} t^{2}}{4}-\frac{t^{4}}{24}$

$p^{1}: u_{1}(x, t)=\frac{x^{2} t^{2}}{4}+\frac{t^{6}\left(3 x^{2}-7\right)}{720}+\frac{t^{8}}{2688}-\frac{x^{4}\left(t^{2}-1\right)}{24}$,

$p^{2}: u_{2}(x, t)=\frac{x^{2} t^{4}}{24}-\frac{t^{6}\left(8 x^{2}-7\right)}{720}+\frac{t^{8}\left(36 x^{2}-64\right)}{40320}$

$$
-\frac{t^{10}\left(168 x^{2}-519\right)}{3628800}-\frac{43 t^{12}}{1064480}
$$

Proceeding in a similar manner and the solution is $u(x, t)=u_{0}(x, t)+u_{1}(x, t)+u_{2}(x, t)+\cdots$

$$
\begin{gathered}
u(x, t)=\frac{t^{2}}{2}+\frac{x^{2}}{2}-\frac{t^{4}}{24}-\frac{t^{4}\left(x^{2}-1\right)}{24}+\frac{t^{6}\left(3 x^{2}-7\right)}{720} \\
+\frac{t^{8}}{2688}+\cdots
\end{gathered}
$$

This is the approximate solution of equation (1) with condition (2) by LTHPM. The HPM approximate analytical solution of equation (1) is [2]

$u(x, t)=\frac{x^{2}}{2}+\frac{t^{2}\left(6 x^{2}+t^{2}\right)}{24}+t^{6}\left(\frac{x^{2}}{240}+\frac{t^{2}}{2688}\right)+\cdots$

Table 1 and Table 2 shows the comparison between the numerical results obtained by HPM, LTHPM and exact solution of equation (1) for $t=0.5$ and $x=0.5$ respectively and its graphical representation is shown in the Fig. -1 and Fig.-2 respectively. It shows that result obtained from LTHPM has a good agreement with the exact solution in comparison with HPM.

\subsection{Illustration II}

Consider

$\frac{\partial^{2} u}{\partial t^{2}}-u^{2} \frac{\partial^{2} u}{\partial x^{2}}=2\left(1-\left(x^{2}+t^{2}\right)^{2}\right)$

with the following conditions

$u(x, 0)=x^{2}, u_{t}(x, 0)=0$

Equation (9) has exact solution $u(x, t)=x^{2}+t^{2}$.

According to LTHPM $[3,4,6,7]$, first apply LT on both sides of equation (10),

$L\left\{\frac{\partial^{2} u}{\partial t^{2}}\right\}-L\left\{u^{2} \frac{\partial^{2} u}{\partial x^{2}}\right\}=2 L\left\{1-\left(x^{2}+t^{2}\right)^{2}\right\}$

Using the LT properties and with the help of specified initial condition (10), equation (11) can be written as

$L[u(x, t)]=\frac{x^{2}}{s}+\frac{2}{s^{3}}-\frac{2 x^{4}}{s^{3}}-\frac{8 x^{2}}{s^{5}}-\frac{48}{s^{7}}+\frac{1}{s^{2}} L\left[u^{2} \frac{\partial^{2} u}{\partial x^{2}}\right]$

Using inverse LT to equation (12), we get,

$$
\begin{array}{r}
u(x, t)=x^{2}+t^{2}-x^{4} t^{2}-\frac{x^{2} t^{4}}{3}-\frac{t^{6}}{15} \\
+L^{-1}\left\{\frac{1}{s^{2}} L\left[u^{2} \frac{\partial^{2} u}{\partial x^{2}}\right]\right\}
\end{array}
$$

Applying the HPM, according to which $u$ is expanded into infinite series and nonlinear term is replaced by He's polynomial

$$
\begin{aligned}
\sum_{n=0}^{\infty} p^{n} u_{n}(x, t)= & x^{2}+t^{2}-x^{4} t^{2}-\frac{x^{2} t^{4}}{3}-\frac{t^{6}}{15} \\
& +p\left\{L^{-1}\left[\frac{1}{s^{2}} L\left[\sum_{n=0}^{\infty} p^{n} H_{n}(u)\right]\right]\right\}
\end{aligned}
$$

Equate the coefficient of like powers of $p$ into equation (14) and the required solution of is given by

$u(x, t)=u_{0}(x, t)+u_{1}(x, t)+u_{2}(x, t)+\cdots$

$u(x, t)=x^{2}+t^{2}-x^{6} t^{4}-\frac{3 x^{2} t^{8}}{14}-\frac{37 x^{4} t^{6}}{5}-\frac{t^{10}}{135}$

This is the approximate solution of equation (9) and which is obtained by considering first two approximations only. The HPM solution of equation (9) is [2]

$$
u(x, t)=x^{2}-\frac{t^{2}\left(15 x^{4}+5 t^{2} x^{2}+t^{4}\right)}{15}-t^{8}\left(\frac{t^{2}}{135}+\frac{3 x^{2}}{14}\right)
$$$$
+\cdots
$$

Comparison between the numerical results obtained by HPM, LTHPM and exact solution of equation (9) is shown in the following Table 3 and Table 4 and Fig.-3and Fig.-4. It shows that the solution obtained by LTHPM has a good agreement with the exact solution in comparison with HPM.

\section{CONCLUSIONS}

In the present paper the application of LTHPM is shown for two nonlinear differential equations and compared with 
available exact solution. On comparing the obtained solution by LTHPM with the exact solution, it is found that it matches well with the exact solution in each case. The present method is reliable, elegant and yields the solution in a rapidly convergent sequence. This proves that the present
method-LTHPM is a powerful mathematical method for solving non-linear equations and very effective and quite accurate. Hence, LTHPM can be conveniently used in solving nonlinear pde arising in different fields of sciences and engineering.

Table -1: HPM, LTHPM and Exact solution comparison at $t=0.5$

\begin{tabular}{|l|l|l|l|l|l|}
\hline & Exact Solution & HPM Solution & HPM Error & LTHPM Solution & LTHPM Error \\
\hline $\boldsymbol{x}=\mathbf{0 . 0}$ & 0.125 & 0.18750145 & -0.06250145 & 0.1248539 & 0.000146097 \\
\hline $\boldsymbol{x}=\mathbf{0 . 1}$ & 0.13 & 0.2025021 & -0.0725021 & 0.12982851 & 0.000171487 \\
\hline $\boldsymbol{x}=\mathbf{0 . 2}$ & 0.145 & 0.24750406 & -0.10250406 & 0.14475234 & 0.000247659 \\
\hline $\boldsymbol{x}=\mathbf{0 . 3}$ & 0.17 & 0.32250731 & -0.15250731 & 0.16962539 & 0.000374612 \\
\hline $\boldsymbol{x}=\mathbf{0 . 4}$ & 0.205 & 0.42751187 & -0.22251187 & 0.20444765 & 0.000552347 \\
\hline $\boldsymbol{x}=\mathbf{0 . 5}$ & 0.25 & 0.56251773 & -0.31251773 & 0.24921914 & 0.000780862 \\
\hline $\boldsymbol{x}=\mathbf{0 . 6}$ & 0.305 & 0.72752489 & -0.42252489 & 0.30393984 & 0.001060159 \\
\hline $\boldsymbol{x}=\mathbf{0 . 7}$ & 0.37 & 0.92253335 & -0.55253335 & 0.36860976 & 0.001390237 \\
\hline $\boldsymbol{x}=\mathbf{0 . 8}$ & 0.445 & 1.14754312 & -0.70254312 & 0.4432289 & 0.001771097 \\
\hline $\boldsymbol{x}=\mathbf{0 . 9}$ & 0.53 & 1.40255419 & -0.87255419 & 0.52779726 & 0.002202737 \\
\hline $\boldsymbol{x}=\mathbf{1 . 0}$ & 0.625 & 1.68756656 & -1.06256656 & 0.62231484 & 0.002685159 \\
\hline
\end{tabular}

Table -2: HPM, LTHPM and Exact solution comparison at $x=0.5$

\begin{tabular}{|l|l|l|l|l|l|}
\hline & Exact Solution & HPM Solution & HPM Error & LTHPM Solution & LTHPM Error \\
\hline $\boldsymbol{t}=\mathbf{0 . 0}$ & 0.125 & 0 & 0.125 & 0.125 & 0 \\
\hline $\boldsymbol{t}=\mathbf{0 . 1}$ & 0.13 & 0.0201 & 0.1099 & 0.12999895 & $1.04998 \mathrm{E}-06$ \\
\hline $\boldsymbol{t}=\mathbf{0 . 2}$ & 0.145 & 0.08160007 & 0.06339993 & 0.1449828 & $1.71984 \mathrm{E}-05$ \\
\hline $\boldsymbol{t}=\mathbf{0 . 3}$ & 0.17 & 0.18810078 & -0.01810078 & 0.16990957 & $9.04319 \mathrm{E}-05$ \\
\hline $\boldsymbol{t}=\mathbf{0 . 4}$ & 0.205 & 0.34560451 & -0.14060451 & 0.2046993 & 0.000300698 \\
\hline $\boldsymbol{t}=\mathbf{0 . 5}$ & 0.25 & 0.56251773 & -0.31251773 & 0.24921914 & 0.000780862 \\
\hline $\boldsymbol{t}=\mathbf{0 . 6}$ & 0.305 & 0.84965485 & -0.54465485 & 0.30326236 & 0.001737643 \\
\hline $\boldsymbol{t}=\mathbf{0 . 7}$ & 0.37 & 1.220244 & -0.850244 & 0.36652147 & 0.003478532 \\
\hline $\boldsymbol{t}=\mathbf{0 . 8}$ & 0.445 & 1.68993548 & -1.24493548 & 0.4385553 & 0.006444698 \\
\hline $\boldsymbol{t}=\mathbf{0 . 9}$ & 0.53 & 2.27681373 & -1.74681373 & 0.51875013 & 0.011249869 \\
\hline $\boldsymbol{t}=\mathbf{1 . 0}$ & 0.625 & 3.00141369 & -2.37641369 & 0.6062748 & 0.018725198 \\
\hline
\end{tabular}

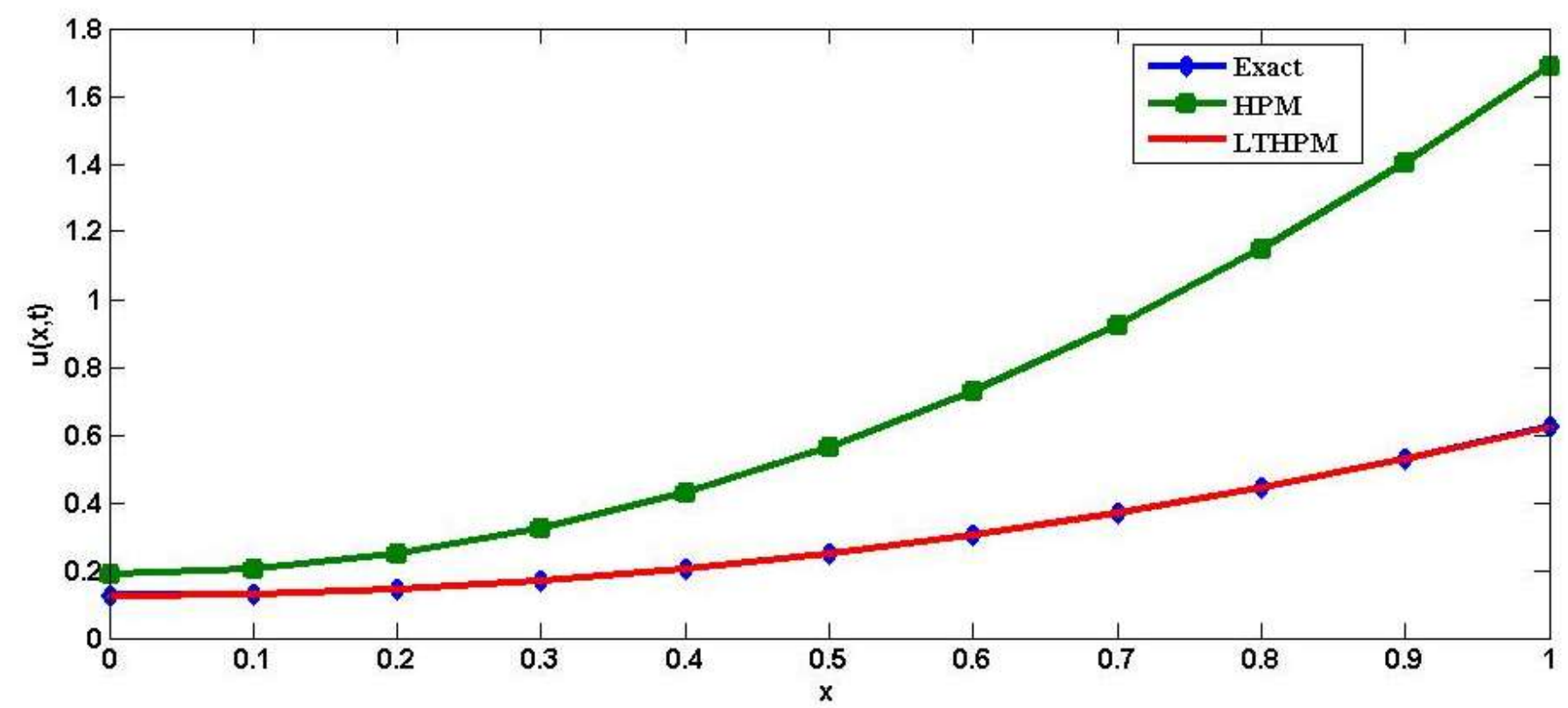

Fig -1: Graphical representation of comparison of HPM, LTHPM and Exact solution at $t=0.5$ 


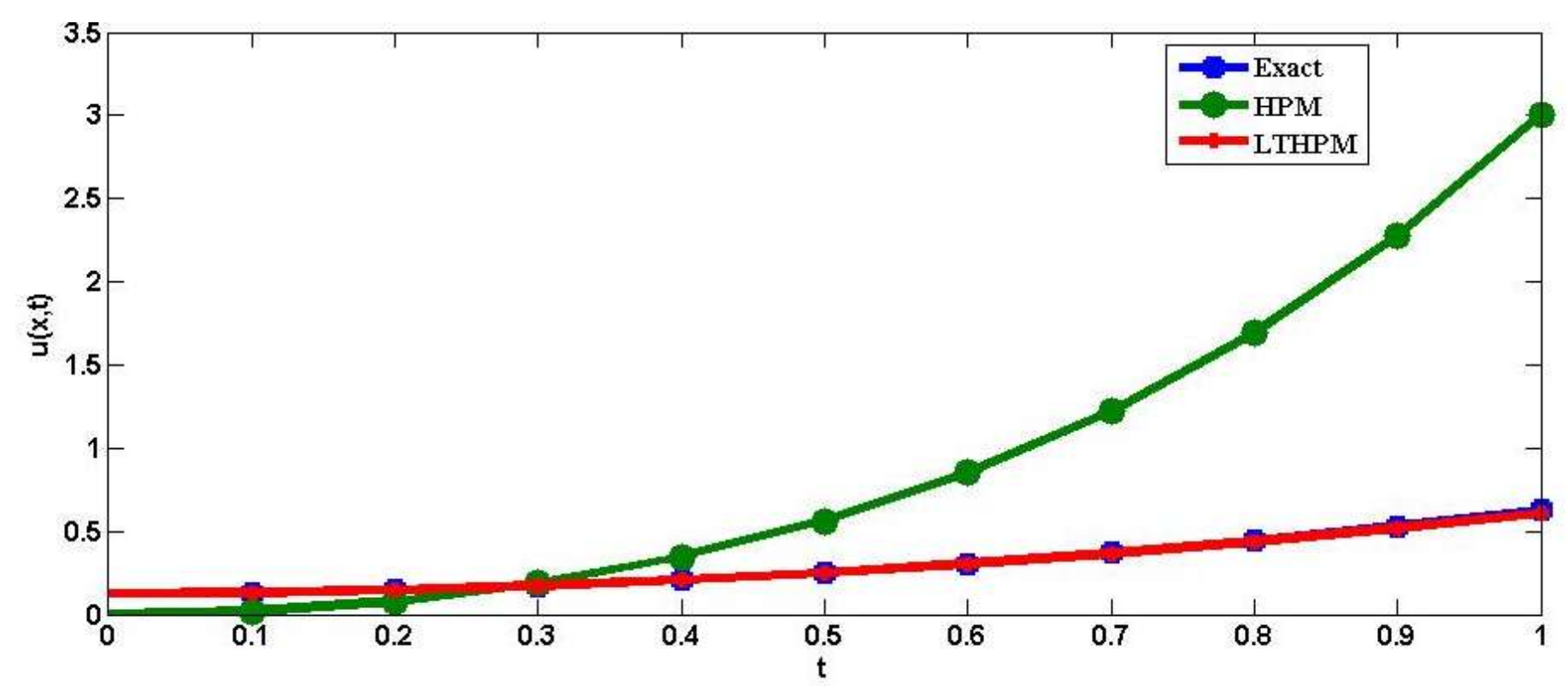

Fig -2: Graphical representation comparison of HPM, LTHPM and Exact solution at $x=0.5$

Table -3: HPM, LTHPM and Exact solution comparison at $t=0.5$

\begin{tabular}{|l|l|l|l|l|l|}
\hline & Exact Solution & HPM Solution & HPM Error & LTHPM Solution & LTHPM Error \\
\hline $\boldsymbol{x}=\mathbf{0 . 0}$ & 0.25 & 0.2489511 & 0.0010489 & 0.24999277 & $7.2338 \mathrm{E}-06$ \\
\hline $\boldsymbol{x}=\mathbf{0 . 1}$ & 0.26 & 0.2487094 & 0.0112906 & 0.25998305 & $1.6952 \mathrm{E}-05$ \\
\hline $\boldsymbol{x}=\mathbf{0 . 2}$ & 0.29 & 0.24768428 & 0.04231572 & 0.28993473 & $6.5271 \mathrm{E}-05$ \\
\hline $\boldsymbol{x}=\mathbf{0 . 3}$ & 0.34 & 0.24497576 & 0.09502424 & 0.33976781 & 0.00023219 \\
\hline $\boldsymbol{x}=\mathbf{0 . 4}$ & 0.41 & 0.23908384 & 0.17091616 & 0.40927395 & 0.00072605 \\
\hline $\boldsymbol{x}=\mathbf{0 . 5}$ & 0.5 & 0.2279085 & 0.2720915 & 0.49800399 & 0.00199601 \\
\hline $\boldsymbol{x}=\mathbf{0 . 6}$ & 0.61 & 0.20874976 & 0.40125024 & 0.60511043 & 0.00488957 \\
\hline $\boldsymbol{x}=\mathbf{0 . 7}$ & 0.74 & 0.17830761 & 0.56169239 & 0.72914493 & 0.01085507 \\
\hline $\boldsymbol{x}=\mathbf{0 . 8}$ & 0.89 & 0.13268205 & 0.75731795 & 0.86781083 & 0.02218917 \\
\hline $\boldsymbol{x}=\mathbf{0 . 9}$ & 1.06 & 0.06737309 & 0.99262691 & 1.01767063 & 0.04232937 \\
\hline $\boldsymbol{x}=\mathbf{1 . 0}$ & 1.25 & -0.02271929 & 1.27271929 & 1.17380849 & 0.07619151 \\
\hline
\end{tabular}

Table -4: HPM, LTHPM and Exact solution comparison at $x=0.5$

\begin{tabular}{|l|l|l|l|l|l|}
\hline & Exact Solution & HPM Solution & HPM Error & LTHPM Solution & LTHPM Error \\
\hline $\boldsymbol{t}=\mathbf{0 . 0}$ & 0.25 & 0 & 0.25 & 0.25 & 0 \\
\hline $\boldsymbol{t}=\mathbf{0 . 1}$ & 0.26 & 0.0093666 & 0.2506334 & 0.25999839 & $1.6144 \mathrm{E}-06$ \\
\hline $\boldsymbol{t}=\mathbf{0 . 2}$ & 0.29 & 0.03736226 & 0.25263774 & 0.28997157 & $2.8427 \mathrm{E}-05$ \\
\hline $\boldsymbol{t}=\mathbf{0 . 3}$ & 0.34 & 0.08364784 & 0.25635216 & 0.33983242 & 0.00016758 \\
\hline $\boldsymbol{t}=\mathbf{0 . 4}$ & 0.41 & 0.14755771 & 0.26244229 & 0.40935363 & 0.00064637 \\
\hline $\boldsymbol{t}=\mathbf{0 . 5}$ & 0.5 & 0.2279085 & 0.2720915 & 0.49800399 & 0.00199601 \\
\hline $\boldsymbol{t}=\mathbf{0 . 6}$ & 0.61 & 0.32264502 & 0.28735498 & 0.60463282 & 0.00536718 \\
\hline $\boldsymbol{t}=\mathbf{0 . 7}$ & 0.74 & 0.42822587 & 0.31177413 & 0.72690506 & 0.01309494 \\
\hline $\boldsymbol{t}=\mathbf{0 . 8}$ & 0.89 & 0.53860724 & 0.35139276 & 0.86034555 & 0.02965445 \\
\hline $\boldsymbol{t}=\mathbf{0 . 9}$ & 1.06 & 0.64362705 & 0.41637295 & 0.99679473 & 0.06320527 \\
\hline $\boldsymbol{t}=\mathbf{1 . 0}$ & 1.25 & 0.72652116 & 0.52347884 & 1.12200728 & 0.12799272 \\
\hline
\end{tabular}




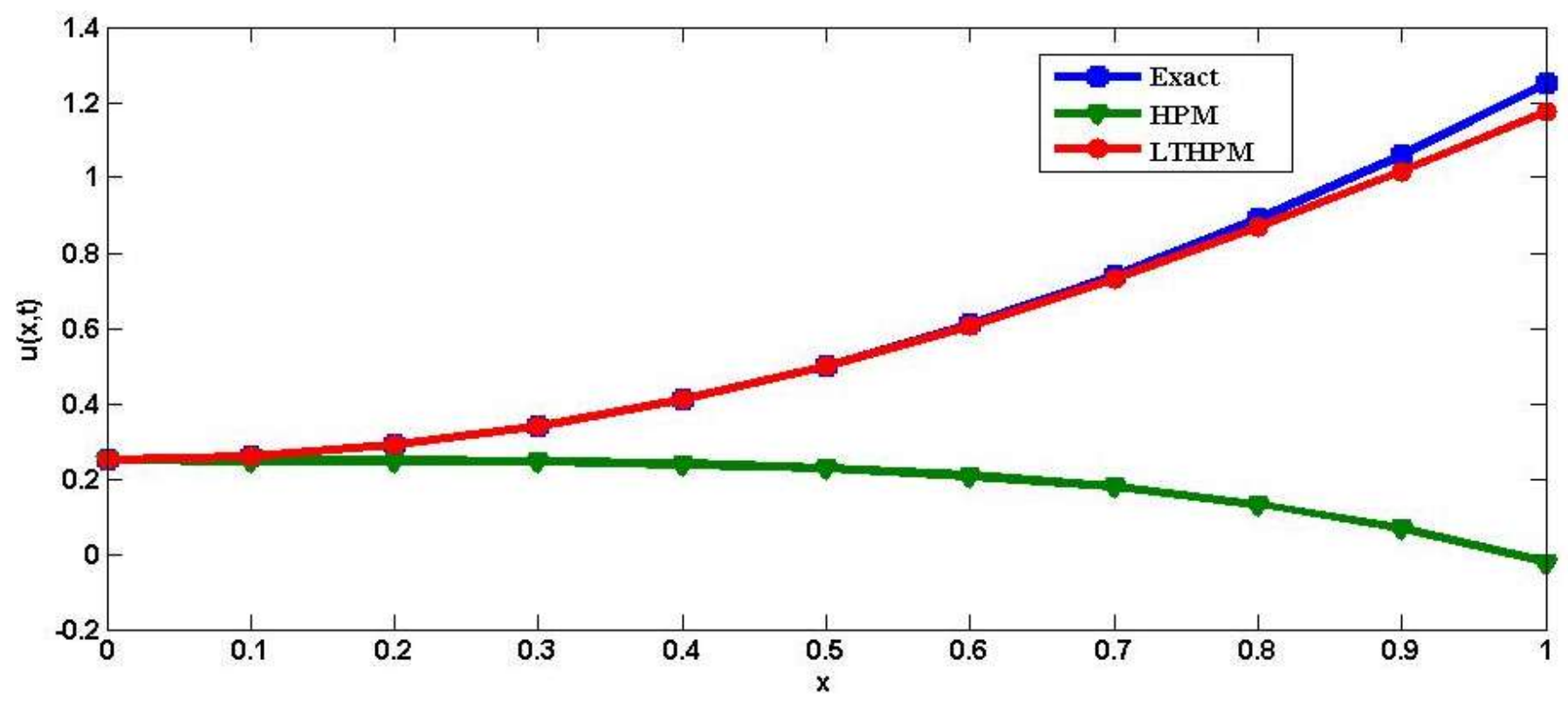

Fig -3: Graphical representation of comparison of HPM, LTHPM and Exact solution at $t=0.5$

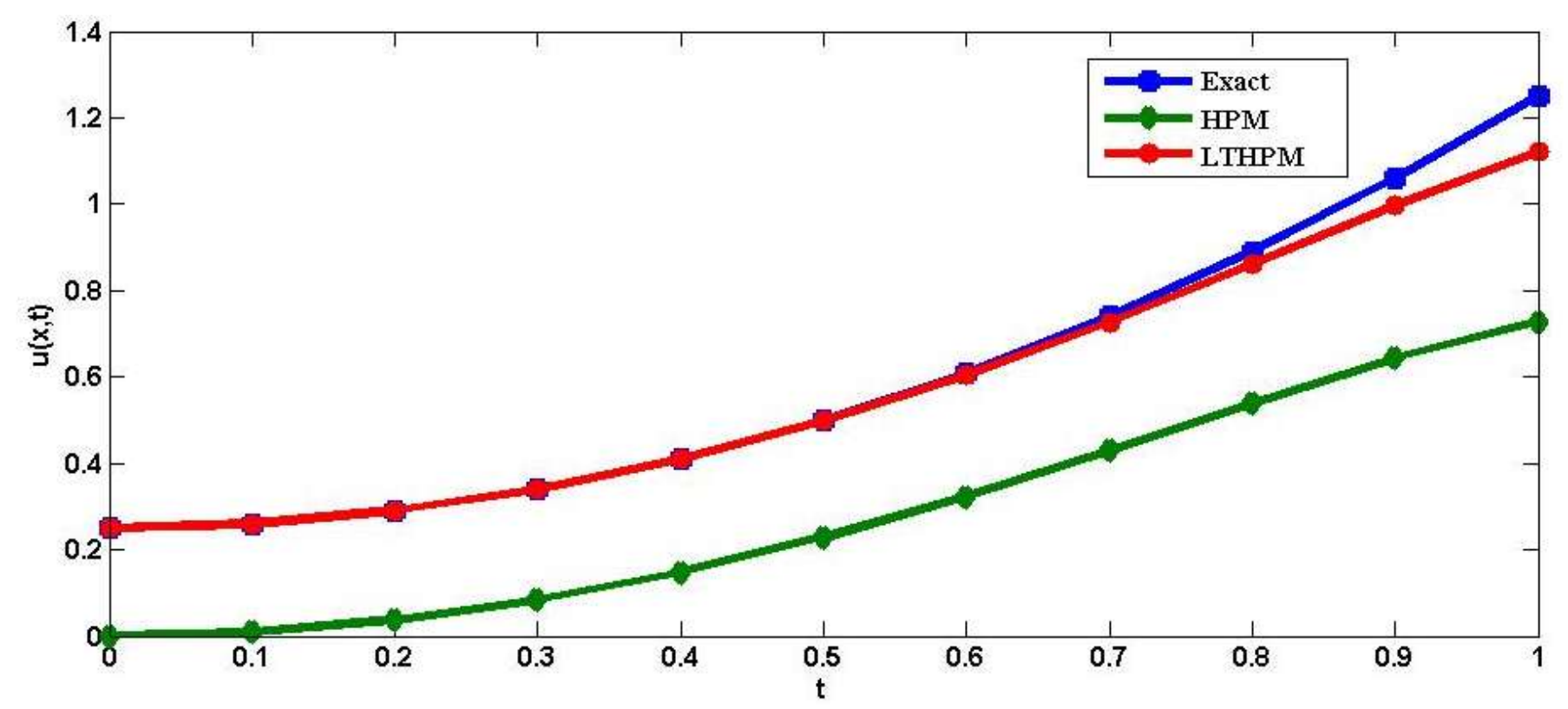

Fig -4: Graphical representation comparison of HPM, LTHPM and Exact solution at $x=0.5$

\section{REFERENCES}

[1]. A. Sadighi, D.D. Ganji, (2007), Exact solutions of Laplace equation by homotopy-perturbation and Adomian decomposition methods, Physics Letters A $367,83-87$.

[2]. B. Ghazanfari, A.G. Ghazanfari and M. Fuladvanda (2011), Modification of the Homotopy Perturbation Method for Numerical Solution of Nonlinear Wave and System of Nonlinear Wave Equations, The Journal of Mathematics and Computer Science, 3(2), 212- 224.

[3]. Hradyesh Kumar Mishra, Atulya K. Nagar (2012), HeLaplace Method for Linear and Nonlinear Partial Differential Equations, Journal of Applied Mathematics, Hindawi Publishing Corporation, Volume 2012, Article ID 180315,16 pages.
[4]. Jagdev Singh, Devendra Kumar and Sushila (2012), homotopy perturbation algorithm using laplace transform for gas dynamics equation, Journal of the Applied Mathematics, Statistics and Informatics (JAMSI), 8(1), 55-61.

[5]. Ji-Huan He (1999), Homotopy perturbation technique, Comput. Methods Appl. Mech. Engrg. 178, 257 262 .

[6]. Sumit Gupta, Devendra Kumar and Jagdev Singh (2015), Analytical solutions of convection-diffusion problems by combining Laplace transform method and homotopy perturbation method, Alexandria Engineering Journal, 54, 645-651.

[7]. Yasir Khan, Qingbiao Wu (2011), Homotopy perturbation transform method for nonlinear equations using He's polynomials, Computers and Mathematics with Applications, 61, 1963-1967. 